\title{
ARTICLE
}

\section{The impact of lifestyle intervention on sedentary time in individuals at high risk of diabetes}

\author{
Bonny Rockette-Wagner ${ }^{1}$ - Sharon Edelstein ${ }^{2} \cdot$ Elizabeth M. Venditti $^{1} \cdot$ Deepti Reddy $^{2}$. \\ George A. Bray ${ }^{3}$ - Mary Lou Carrion-Petersen ${ }^{4}$ - Dana Dabelea ${ }^{5,14}$. \\ Linda M. Delahanty ${ }^{6,15}$ - Hermes Florez ${ }^{7,16}$ • Paul W. Franks ${ }^{8,9,10}$ • Maria G. Montez ${ }^{11}$ • \\ Richard Rubin $^{12}$ - Andrea M. Kriska ${ }^{1,13}$ • for the Diabetes Prevention Program Research \\ Group
}

Received: 29 October 2014 / Accepted: 3 March 2015 / Published online: 8 April 2015

(C) Springer-Verlag Berlin Heidelberg 2015

\begin{abstract}
Aims/hypothesis The Diabetes Prevention Program (DPP) lifestyle intervention successfully achieved its goal of increasing leisure physical activity levels. This current study examines whether the lifestyle intervention also changed time spent being sedentary and the impact of sedentary time on diabetes development in this cohort.

Methods 3,232 DPP participants provided baseline data. Sedentary behaviour was assessed via an intervieweradministered questionnaire and reported as time spent watching television specifically (or combined with sitting at

Richard Rubin died on 25 March 2013.

A complete list of Diabetes Prevention Program centres, investigators and staff can be found in the electronic supplementary material (ESM investigators).
\end{abstract}

Electronic supplementary material The online version of this article (doi:10.1007/s00125-015-3565-0) contains peer-reviewed but unedited supplementary material, which is available to authorised users.

Andrea M. Kriska

dppmail@bsc.gwu.edu

1 Department of Epidemiology, University of Pittsburgh, Pittsburgh, PA, USA

2 George Washington University, Washington, DC, USA

3 Pennington Biomedical Research Center, Baton Rouge, LA, USA

4 University of California, San Diego, CA, USA

5 University of Colorado, Denver, CO, USA

6 Massachusetts General Hospital, Boston, MA, USA

7 University of Miami, Miami, FL, USA

8 Department of Clinical Science, Genetic and Molecular Epidemiology Unit, Lund University, Malmö, Sweden work). Mean change in sedentary time was examined using repeated measures ANCOVA. The relationship between sedentary time and diabetes incidence was determined using Cox proportional hazards models.

Results During the DPP follow-up (mean: 3.2 years), sedentary time declined more in the lifestyle than the metformin or placebo participants $(p<0.05)$. For the lifestyle group, the decrease in reported mean television watching time $(22$ [95\% CI $26,17] \mathrm{min} /$ day) was greater than in the metformin or placebo groups $(p<0.001)$. Combining all participants together, there was a significantly increased risk of developing diabetes with

$$
\text { University, Umeå, Sweden }
$$

11 University of Texas Health Science Center, San Antonio, TX, USA

12 Johns Hopkins School of Medicine, Baltimore, MD, USA

13 DPP Coordinating Center, The George Washington University, Biostatistics Center, 6110 Executive Boulevard, Suite 750, Rockville, MD 20852-3943, USA

14 Colorado School of Public Health, University of Colorado, Denver, CO, USA

15 Department of Medicine, Harvard Medical School, Boston, MA, USA

16 University of Miami/VA-GRECC, Miami, FL, USA 
increased television watching (3.4\% per hour spent watching television), after controlling for age, sex, treatment arm and leisure physical activity $(p<0.01)$, which was attenuated when time-dependent weight was added to the model.

Conclusions/interpretation In the DPP, the lifestyle intervention was effective at reducing sedentary time, which was not a primary goal. In addition, in all treatment arms, individuals with lower levels of sedentary time had a lower risk of developing diabetes. Future lifestyle intervention programmes should emphasise reducing television watching and other sedentary behaviours in addition to increasing physical activity. Trial registration: ClinicalTrials.gov NCT00004992

Keywords Diabetes Prevention Program - Sedentary behaviour. Television watching . Type 2 diabetes

$\begin{array}{ll}\text { Abbreviations } \\ \text { DPP } & \text { Diabetes Prevention Program } \\ \text { DPPOS } & \text { Diabetes Prevention Program Outcomes Study } \\ \text { MAQ } & \text { Modifiable Activity Questionnaire } \\ \text { MET } & \text { Metabolic equivalent } \\ \text { TV } & \text { Television }\end{array}$

\section{Introduction}

Sedentary behaviour has been described as time spent in activities with low energy expenditure such as watching television (TV), using the computer, or sitting during commuting and while working [1], and has been identified as a potential risk factor for diabetes and the metabolic syndrome, independent of physical activity [1-4]. To date, most lifestyle interventions designed to decrease diabetes risk have focused on weight loss, dietary change and increasing physical activity levels, but have not examined the contribution of sedentary time [5].

The activity goal in the lifestyle arm of the Diabetes Prevention Program (DPP) was to achieve at least $150 \mathrm{~min} /$ week of moderate to vigorous activity and was met by the majority of the lifestyle participants [5]. What is not known is whether the intervention effort had any impact on time spent being sedentary. Previous results suggest that interventions focused on increasing physical activity may actually lead to increases in sitting time [6]. This manuscript examines whether the DPP lifestyle intervention, which was shown to be effective at increasing physical activity, also improved selfreported sitting time. The effect of sedentary behaviour on diabetes development was also examined.

\section{Methods}

Design and participants The DPP was a multicentre, randomised controlled clinical trial designed to determine whether metformin or lifestyle intervention could prevent or delay type 2 diabetes in adults at high risk of developing the disease [7]. When the DPP results were reported (mean follow-up: 2.8 years), diabetes incidence was reduced in the metformin and lifestyle intervention arms compared with placebo by $31 \%$ and $58 \%$, respectively [ 8$]$. The final data collection for the formal DPP study took slightly longer (3.2 years) and is the follow-up time of this current effort [9].

The study design, methods and primary results for the DPP have been published [7, 8]. Briefly, the study enrolled 3,234 overweight US adults (1996-1999) of at least 25 years of age. Informed consent was obtained for all participants and the study was approved by the institutional review boards of each institution.

The lifestyle intervention has been previously described [5]. The goals were to achieve a 7\% weight loss and at least $150 \mathrm{~min} /$ week of moderate intensity activity (e.g. brisk walking) and included behavioural self-management strategies such as self-monitoring. Although reduction in sedentary time was not a primary goal of the intervention, suggestions for limiting inactive lifestyle choices (e.g. reducing TV watching time) were discussed briefly in the curriculum and encouraged occasionally throughout the programme.

Measurements Demographic information was collected prior to randomisation [7]. Weight was collected at baseline and semi-annually [8]. Incident diabetes was identified annually using a $75 \mathrm{~g}$ OGTT and semi-annually using fasting glucose levels, with values confirmed within 6 weeks [7].

Self-reported past year physical activity and sedentary time was collected at baseline and annually via the intervieweradministered Modifiable Activity Questionnaire (MAQ). The MAQ was designed to collect both leisure and work-related, primarily moderate to vigorous activity and has been previously validated against other instruments including accelerometers [10-13].

Sedentary time was determined from the MAQ as average daily time spent watching TV and time spent sitting at work. Supporting the validation study by Jacobi et al [13], we validated the sedentary questions on the MAQ in a subsample of the DPP study population $(n=695)$ at year 10 of the Diabetes Prevention Program Outcomes Study (DPPOS) using ActiGraph GT3X triaxial accelerometers (ActiGraph, Pensacola, FL, USA). Since individual activities cannot be identified from accelerometer data, total time spent sedentary (defined as $<150$ counts/min [14]) was compared with reported time spent TV watching and reported time spent TV watching plus work sitting from the MAQ using Spearman rank order correlations.

TV watching typically accounts for a small portion of the time spent in total sedentary pursuits recorded by accelerometers 
$[13,15,16]$. Therefore, we would not anticipate high correlations between reported TV watching and total sedentary time from accelerometers and our results support this hypothesis (partial $\rho=0.11, p=0.0037$; controlling for age and sex). As also expected, adding another component of sedentary behaviour (reported time spent sitting at work) to TV watching from the MAQ strengthened the correlation ( partial $\rho=0.37, p<0.0001$ ). Similar correlations between reported total sitting time from questionnaires and total sedentary time from accelerometers have been published for other validated questionnaires including the International Physical Activity Questionnaire (IPAQ; $\rho=$ 0.17-0.23), the Global Physical Activity Questionnaire (GPAQ; $\rho=-0.02$ to 0.40 ) and Past Year Total Physical Activity Questionnaire (PYTPAQ; $\rho=0.32$ ) [17].

Statistical methods Analyses were conducted using SAS (v. 9.2, SAS Institute, Cary, NC, USA). The relationship between average time spent watching TV and leisure physical activity in metabolic equivalent (MET [h/week], averaged over intervention years 1-3) was expressed using Spearman correlations. ANOVA/ANCOVA was used to compare mean baseline and yearly change (controlling for baseline) values across the three treatment groups for minutes of reported TV watching and combined sedentary activity (TV plus time sitting at work). Repeated measures ANOVA was used to assess change from baseline to end of study within treatment groups and subgroups based on demographic variables, body composition, working status and meeting lifestyle goals. In these analyses, age categories ( $<40,40$ to $<50,50$ to $<60$ and $\geq 60$ years), race/ethnicity (white non-Hispanic and other), baseline BMI $\left(\geq 30 \mathrm{~kg} / \mathrm{m}^{2}\right.$ and $<30 \mathrm{~kg} / \mathrm{m}^{2}$ ) and work status (worked at home or worked outside the home) were used.

The effect of TV watching and combined sedentary activity on diabetes incidence was examined using Cox proportional hazards models with time-dependent covariates. Owing to the lack of an interaction by treatment arm, Cox models were examined for both the entire cohort (controlling for treatment) and the lifestyle arm alone. All models were adjusted for baseline age and sex. Additional models were adjusted for timedependent covariates including leisure physical activity in MET (h/week) from the MAQ and body weight.

\section{Results}

Overall, 3,232 participants completed the MAQ at baseline. Spearman correlations showed a modest, inverse relationship between TV watching time and leisure physical activity $(-0.13, p<0.0001)$, even after controlling for age and sex $(-0.15, p<0.0001)$. Mean values for baseline TV watching time were not significantly different $(p=0.49$ ) across the three treatment groups: placebo was 144 (95\% CI 138, 150), metformin was $139(95 \%$ CI 133, 145) and lifestyle was 144 (95\%
CI 138,151$) \mathrm{min} /$ day. Additionally, the combination variable (TV plus time sitting at work) at baseline was not significantly different ( $p=0.25$ ) across the three treatment arms: 412 (95\% CI 399, 423), 410 (95\% CI 399, 421) and 423 (95\% CI 410, 435) $\mathrm{min} /$ day for the placebo, metformin and lifestyle groups, respectively.

Figure 1 illustrates the mean change from baseline in selfreported TV watching by study year for each treatment group. Mean change in reported TV watching between baseline and year 1 for the placebo, metformin and lifestyle groups were -7 $(95 \% \mathrm{CI}-13,-2),-3(95 \% \mathrm{CI}-8,2)$ and $-22(95 \% \mathrm{CI}-28$, $-17) \mathrm{min} /$ day, respectively. Differences between treatment groups for change in TV watching from baseline were significant $(p<0.001)$ at each follow-up year, with consistently greater reductions in the lifestyle group than both the placebo and metformin groups. Overall, mean time spent watching TV decreased significantly during follow-up (mean: 3.2 years) in the lifestyle $(-22[95 \% \mathrm{CI}-26,-17] \mathrm{min} /$ day $)$ and placebo $(-8[95 \% \mathrm{CI}-12,-3] \mathrm{min} /$ day $)$ groups, but not in the metformin group $(-3[95 \% \mathrm{CI}-7,1] \mathrm{min} /$ day $)$, with significantly greater reductions in TV watching in lifestyle participants than either metformin or placebo participants $(p<0.05)$.

For the lifestyle participants, this reduction in reported TV watching time throughout follow-up was observed for all participant subgroups including age, sex, work status, race/ethnicity, obesity status, or those achieving the weight and/or activity goal(s) (data not shown). Similarly, the lifestyle group had the greatest reduction in mean time spent in the combination sedentary variable (TV watching and time sitting at work) during follow-up. Repeated measures change was -9 (95\% CI $-17,-1),-6(95 \% \mathrm{CI}-14,2)$ and -37 (95\% CI -44 , -28 ) $\mathrm{min} /$ day for the placebo, metformin and lifestyle groups, respectively ( $p<0.001$ for differences between groups).

The impact of time-varying sedentary behaviour on diabetes incidence was then investigated (Table 1). For participants in all treatment arms, the risk of developing diabetes increased approximately $3.4 \%$ (HR 1.034 [95\% CI 1.004, 1.065], $p=$ 0.026 ) for each hour spent watching TV after adjustment for

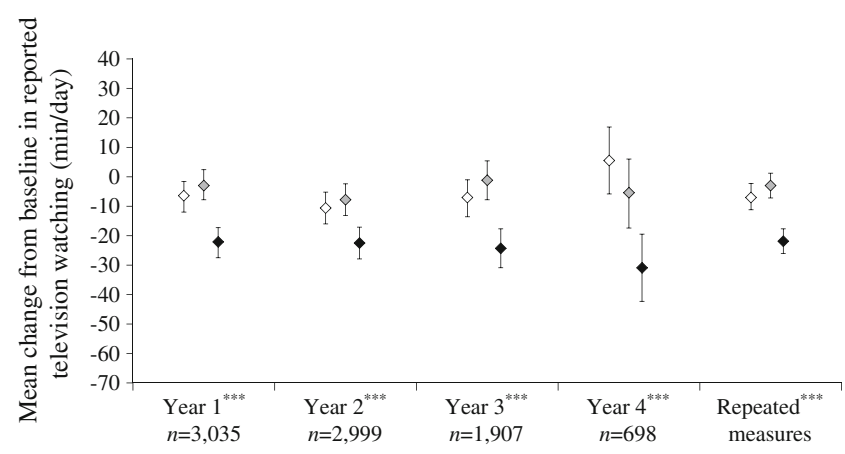

Fig. 1 Mean $(95 \% \mathrm{CI})$ change from baseline in TV watching by treatment group during DPP follow-up (white, placebo; grey, metformin; black, lifestyle). ${ }^{* * *} p<0.001$ (ANOVA) for comparison between treatment groups 
Table 1 HR $(95 \% \mathrm{CI})$ for diabetes development in all DPP participants for each hour spent in sedentary behaviour(s)

\begin{tabular}{llllll}
\hline Model & HR $(95 \% \mathrm{CI})$ per hour of TV watching & & \multicolumn{2}{l}{$\begin{array}{l}\text { HR }(95 \% \mathrm{CI}) \text { per hour of combined } \\
\text { sedentary activity (TV + sitting at work) }\end{array}$} \\
\cline { 2 - 3 } & $\begin{array}{l}\text { Lifestyle arm } \\
(n=1,077)\end{array}$ & $\begin{array}{l}\text { All participants } \\
(n=3,232)\end{array}$ & & $\begin{array}{l}\text { Lifestyle arm } \\
(n=1,077)\end{array}$ & $\begin{array}{l}\text { All participants } \\
(n=3,232)\end{array}$ \\
\hline Model 1: controlling for age and sex & $1.047(0.988,1.109)$ & $1.036(1.006,1.067)$ & $1.019(0.987,1.051)$ & $1.028(1.011,1.045)$ \\
Model 2: controlling for age, sex, and $\mathrm{PA}^{\mathrm{b}, \mathrm{c}}$ & $1.038(0.979,1.101)$ & $1.034(1.004,1.065)$ & $1.012(0.981,1.045)$ & $1.026(1.008,1.044)$ \\
Model 3: controlling for age, sex, $\mathrm{PA}^{\mathrm{b}}$ and weight & $1.011(0.950,1.075)$ & $1.021(0.990,1.053)$ & $0.984(0.950,1.020)$ & $1.012(0.994,1.031)$ \\
\hline
\end{tabular}

${ }^{a}$ Controlling for treatment arm

${ }^{\mathrm{b}}$ PA included leisure PA for models with TV watching only and leisure and work-related PA for models with combined leisure and sitting at work

${ }^{\mathrm{c}}$ Time-dependent covariates were used for PA and weight

PA, physical activity

age, sex, treatment arm and time-dependent leisure physical activity in the Cox proportional hazards models. This association was attenuated when time-dependent weight was added to the model (HR 1.021 [95\% CI 0.990, 1.053], $p=0.17$ ). Similar results were found in the lifestyle participants alone. The association between the combination of TV watching and sitting at work and the risk of diabetes development was weaker, but similar to that of TV watching alone.

\section{Discussion}

The DPP lifestyle intervention resulted in significant increases in leisure physical activity levels in individuals at high risk of developing diabetes [5]. This current effort demonstrates that the intervention also had a positive impact on self-reported time spent in sedentary behaviour that was significant across demographic and BMI specific subgroups. Additionally, these results suggest that time spent sedentary was related to diabetes incidence in the DPP study cohort, independent of physical activity levels.

A recent review suggested that improving physical activity through lifestyle intervention may not necessarily have a similar beneficial effect on sedentary time [6]. By contrast, the results of this study showed that an intervention known to improve moderately intense activity did positively affect sedentary time despite a lack of emphasis on decreasing sedentary behaviour.

This effort suggests that modest improvements in domainspecific sedentary time, such as TV watching, may lead to reduced diabetes incidence in individuals at high risk of developing the disease. Controlling for time-dependent body weight attenuated this finding. However, owing to the known association between body weight and sedentary behaviour, one could argue that adjusting for weight may represent over-controlling [18].
Responses to questions regarding time spent watching TV and sitting at work were used to estimate specific segments of total sedentary time and are known to perform reasonably well [1]. These measures do not represent total sedentary time; as many sedentary behaviours are unplanned and/or unstructured, making them hard to recall or quantify subjectively [1]. Therefore, adding objective measures of physical activity to future interventions that can more accurately quantify all contributions of sedentary behaviour is suggested in order to validate these questionnaire-based findings.

Reducing sedentary behaviours was not a specific goal of the DPP intervention and participants were not asked to log the amount of time they spent sitting. Increased emphasis on reducing TV watching and other sedentary behaviours through other behaviour change strategies, such as more direct encouragement and self-monitoring, would be likely to lead to greater reductions in sedentary time. Additional emphasis on reducing sedentary behaviours in lifestyle intervention programmes that are already focused on increasing leisure physical activity levels is merited.

Acknowledgements The Research Group gratefully acknowledges the commitment and dedication of the participants of the DPP and DPPOS.

Funding During the DPPOS, the National Institute of Diabetes and Digestive and Kidney Diseases (NIDDK) of the National Institutes of Health provided funding to the clinical centres and the Coordinating Center for the design and conduct of the study, and collection, management, analysis and interpretation of the data (U01 DK048489). The Southwestern American Indian Centers were supported directly by the NIDDK, including its Intramural Research Program, and the Indian Health Service. The General Clinical Research Center Program, National Center for Research Resources and the Department of Veterans Affairs supported data collection at many of the clinical centres. Funding was also provided by the National Institute of Child Health and Human Development, the National Institute on Aging, the National Eye Institute, the National Heart Lung and Blood Institute, the Office of Research on Women's Health, the National Institute on Minority Health and Health Disparities, the Centers for Disease Control and Prevention 
and the ADA. Bristol-Myers Squibb and Parke-Davis provided additional funding and material support during the DPP, Lipha (Merck-Sante) provided medication and LifeScan Inc. donated materials during the DPP and DPPOS. The opinions expressed are those of the investigators and do not necessarily reflect the views of the funding agencies.

Duality of interest LMD has a financial interest in Omada Health, a company that develops online behaviour change programmes, with a focus on diabetes. The interests of LMD were reviewed and are managed by Massachusetts General Hospital and Partners HealthCare in accordance with their conflict of interest policies. All other authors declare that there is no duality of interest associated with their contribution to this manuscript.

Contribution statement BRW helped to draft the concept and design, aided in data interpretation, and drafted and revised the manuscript. SE and DR assisted with design, conducted data analysis and assisted with article revisions. EMV assisted with design, aided in the interpretation of data, and provided feedback and assisted with article revisions. GB, MLCP, DD, LMD, HF, PWF, MGM and RR assisted with design, aided in the interpretation of data, and provided feedback and assisted with article revisions. AMK helped to draft the concept and design, aided in data interpretation and assisted with article revisions. All authors other than RR had final approval of the submitted manuscript. BRW is responsible for the integrity of this work as a whole.

\section{References}

1. Owen N, Healy GN, Matthews CE, Dunstan DW (2010) Too much sitting: the population health science of sedentary behavior. Exerc Sport Sci Rev 38:105-113

2. Fitzgerald SJ, Kriska AM, Pereira MA, de Courten MP (1997) Associations among physical activity, television watching, and obesity in adult Pima Indians. Med Sci Sports Exerc 29:910-915

3. Hu FB, Leitzmann MF, Stampfer MJ, Colditz GA, Willett WC, Rimm EB (2001) Physical activity and television watching in relation to risk for type 2 diabetes mellitus in men. Arch Intern Med 161:1542-1548

4. Hu FB, Li TY, Colditz GA, Willett WC, Manson JE (2003) Television watching and other sedentary behaviors in relation to risk of obesity and type 2 diabetes mellitus in women. JAMA 289:1785-1791
5. Wing RR, Hamman RF, Bray GA et al (2004) Achieving weight and activity goals among diabetes prevention program lifestyle participants. Obes Res 12:1426-1434

6. Owen N, Sugiyama T, Eakin EE, Gardiner PA, Tremblay MS, Sallis JF (2011) Adults' sedentary behavior determinants and interventions. Am J Prev Med 41:189-196

7. [No authors listed] (1999) The Diabetes Prevention Program. Design and methods for a clinical trial in the prevention of type 2 diabetes. Diabetes Care 22:623-634

8. Knowler WC, Barrett-Connor E, Fowler SE et al (2002) Reduction in the incidence of type 2 diabetes with lifestyle intervention or metformin. N Engl J Med 346:393-403

9. Hamman RF, Wing RR, Edelstein SL et al (2006) Effect of weight loss with lifestyle intervention on risk of diabetes. Diabetes Care 29: 2102-2107

10. Kriska AM (1997) Modifiable activity questionnaire. Med Sci Sports Exerc 29:S73-S78

11. Momenan AA, Delshad M, Sarbazi N, Rezaei Ghaleh N, Ghanbarian A, Azizi F (2012) Reliability and validity of the Modifiable Activity Questionnaire (MAQ) in an Iranian urban adult population. Arch Iran Med 15:279-282

12. Pettee Gabriel K, McClain JJ, Schmid KK, Storti KL, Ainsworth BE (2011) Reliability and convergent validity of the past-week Modifiable Activity Questionnaire. Public Health Nutr 14:435-442

13. Jacobi D, Charles MA, Tafflet M, Lommez A, Borys JM, Oppert JM (2009) Relationships of self-reported physical activity domains with accelerometry recordings in French adults. Eur J Epidemiol 24:171-179

14. Kozey-Keadle S, Libertine A, Lyden K, Staudenmayer J, Freedson PS (2011) Validation of wearable monitors for assessing sedentary behavior. Med Sci Sports Exerc 43:1561-1567

15. Stamatakis E, Coombs N, Rowlands A, Shelton N, Hillsdon M (2014) Objectively-assessed and self-reported sedentary time in relation to multiple socioeconomic status indicators among adults in England: a cross-sectional study. BMJ Open 4:e006034

16. Saleh D, Janssen I (2014) Interrelationships among sedentary time, sleep duration, and the metabolic syndrome in adults. BMC Public Health 14:666

17. Helmerhorst HJ, Wijndaele K, Brage S, Wareham NJ, Ekelund U (2009) Objectively measured sedentary time may predict insulin resistance independent of moderate- and vigorous-intensity physical activity. Diabetes 58:1776-1779

18. Thorp AA, Owen N, Neuhaus M, Dunstan DW (2011) Sedentary behaviors and subsequent health outcomes in adults a systematic review of longitudinal studies, 1996-2011. Am J Prev Med 41:207215 